\title{
Numerical computation of wave breaking times
}

\author{
Ravi Shankar* \\ Faculty Advisor: Sergei Fomin ${ }^{\dagger}$
}

\begin{abstract}
The time of nonlinear wave collapse is computed numerically for the Hopf equation. Previous numerical criteria for locating the time of wave collapse are either computationally prohibitive to implement or give erroneous results. A new criterion for this purpose is developed analytically using asymptotic analysis of the wave shock development shortly after breaking. The criterion defines the wave breaking time as the onset of energy dissipation. This onset results in a singularity in the third derivative of the energy function, the location of which yields the breaking time. A numerical criterion is formulated from this analytical result and tested against the exact analytical value of the breaking time. This is done by first solving the differential equation with a finite difference method. Then, to compensate for numerical error, a moving average method is developed to refine the energy data. The obtained results give visible convergence to the analytical breaking time as the numerical mesh is refined.
\end{abstract}

\section{Introduction}

Nonlinear wave collapse (breaking) arises in several important contexts. First, in the case of water waves (such as those described by the nonlinear shallow-water equations, see Stoker [10]), wave collapse is associated with the time at which a water wave releases its energy and becomes most destructive. This is relevant for the damage that water waves cause to shipyards and beaches and that tsunami waves do to populated coastlines. Second, for the case of high Reynolds number fluid flow (such as for the inviscid Euler equations of fluid mechanics), nonlinear wave collapse (called blow-up in this context) is associated with the onset of turbulence. The mathematical description of this phenomenon is still an open problem [3]. In this study, a numerical method is introduced which has application towards predicting the time at which nonlinear wave collapse occurs. The setting is one-dimensional, but the possibility exists that the approach developed here could be applied to any of the realistic contexts described above.

The first part of this study details the derivation of an analytical criterion for the wave collapse time. A thorough analytical description of wave-collapse was given by Whitham [14] for the one-dimensional nonlinear advection equation, of which the Hopf equation the present paper considers is a special case. Using the method of characteristics, it was shown that the time of wave collapse is characterized by several equivalent properties: (i) the first intersection of the characteristic lines of invariance, (ii) the time when the slope of the wavefront first becomes unbounded, and (iii) when a shock first develops in the wavefront. For the advection equation considered by Whitham [14], these criteria allow for a straightforward analytical determination of the wave breaking time. However, analytical determination is no longer possible for more complicated equations or for equations in more spatial dimensions, and one must resort to numerical methods (a perturbative approach is given in [2]). The translation of these analytical criteria to mathematically correct and readily implementable numerical criteria is therefore essential for practical purposes. In this study, the results of Whitham [14] are used to develop a new analytical criterion for locating the time of wave collapse that will guide the implementation of a numerical criterion.

The next part of this study documents the application of a numerical criterion for the wave collapse time. For linear equations where wave collapse doesn't occur, numerical solutions have been widely studied in various contexts (see e.g. [9]). In contrast, there have only been a few attempts to develop numerical methods for predicting the time of nonlinear wave collapse. The first is a study of the nonlinear shallow water equations by Jeffrey and Russo [5] that compares results of several numerical strategies used to solve this problem. They used two criteria

\footnotetext{
*Department of Mathematics and Statistics, California State University: Chico, Chico, CA 95929, r.shankar997@gmail.com

$\dagger$ Department of Mathematics and Statistics, California State University: Chico, Chico, CA 95929, sfomin@csuchico.edu
} 
for determining breaking times: (a) solving the differential equation with finite difference methods and checking for when the spatial gradient exceeded some pre-assigned large value, and (b) solving the differential equation numerically with the method of characteristics and locating the first intersection of the characteristic lines. While approach (b) was successful, they found approach (a) to be unsuccessful due to the inability to resolve the steep wavefront sufficiently accurately. In fact, it was shown in a particular case by Tran and Dritschel [13] that the criterion used for approach (a) is incorrect and that breaking actually occurs before the wave becomes its steepest. In this study, we solve the differential equations as in approach (a) but develop a more accurate and ultimately successful breaking criterion. In the case of the method of characteristics of approach (b), while being an accurate approach, it is a notoriously complicated procedure to program for nonlinear systems and hasn't been as widely applied or well-developed as explicit finite-difference methods, the latter of which are employed in the present study.

Klein and Roidot [6] applied results of asymptotic Fourier analysis to the location of the breaking time for the Hopf equation. Their criterion for the collapse time was when the singularity in the wave's complex Fourier Transform first intersected the real axis. At this time, the rate of decreasing modulus with increasing wavenumber of the Fourier coefficients changed from exponential to algebraic, indicative of a singularity in the function's spatial derivative. Their method involves first numerically integrating the time evolution equations for the Fourier coefficients, then applying a least-squares fitting of these coefficients' modulus to an asymptotic formula valid for large Fourier wavenumbers. Their computed results for the breaking time agreed with the analytical solution, but their numerical method was computationally costly for the accuracy of their computed breaking times. Their fitting procedure was also rather empirical. We attempted in this study to develop a straightforward numerical procedure implementable on a laptop computer that requires little to no empirical calibration.

\section{Analytical Developments}

This section describes how we arrived at our analytical results, providing (1) a brief overview of the Hopf equation and wave collapse mechanics; (2) an asymptotic analysis of the nonlinear wave structure after breaking; (3) a computation of the wave energy function based upon the asymptotic results; and (4) a moving average method used to compensate for numerical error.

\subsection{Hopf Equation}

The Hopf equation is a simple prototype model for nonlinear wave breaking and one-dimensional turbulence. It arises as the inviscid limit of the Burgers equation or the pressureless Navier-Stokes equation [14]. It can also be obtained from the nonlinear shallow water equations of uniform water depth when one of the two Riemann invariants is constant (i.e. simple-wave motion [10]). The Hopf equation is:

$$
u_{t}+u u_{x}=0
$$

where $u=u(x, t)$ is a velocity in the above settings. For initial data $u(x, 0)=f(x)$, the general solution to (1) is given implicitly by:

$$
\begin{array}{r}
u(x, t)=f(\xi), \\
x=\xi+t u .
\end{array}
$$

We assume the initial data $f$ to be $C^{3}$ and that $\int_{-\infty}^{\infty}|f(x)| \mathrm{d} x<\infty$. Here, $\xi$ parametrizes a characteristic line; because function $u(x, t)$ is constant along a characteristic line, each value of $\xi$ can be associated to a unique function value. The time at which two distinct characteristics obtain the same position $x$ is called the breaking (collapse) time; at this time, $u(x, t)$ becomes a multivalued function. The breaking time $t_{B}>0$ is given by $t_{B}=-1 / f^{\prime}\left(\xi_{B}\right)$, where $\xi_{B}$ is obtained from $f^{\prime}\left(\xi_{B}\right)=\inf _{\xi} f^{\prime}(\xi)$; the function first breaks at a negatively sloping inflection point. The value of the function at this point is $u_{B}=f\left(\xi_{B}\right)$, and the position at which it breaks is $x_{B}=\xi_{B}+u_{B} t_{B}$.

Although, for $t>t_{B}$, the $u(x, t)$ defined by (2) becomes a multivalued function, a weak single-valued solution $u(x, t)$ to (1) can be obtained by imposing a discontinuous jump bridging two characteristics adjoining the multivalued portion of the curve; this discontinuity is called a shock and moves along the shock trajectory $x=s(t)$, where

$s\left(t_{B}\right)=x_{B}$ gives the original position of the shock. The (Rankine-Hugoniot) conditions that define the propagation 
of the shock position $x=s(t)$ are obtained from the condition that conservation of mass be satisfied:

$$
\frac{d}{d t} \int_{-\infty}^{\infty} u(x, t) d x=0
$$

This and other conservation laws such as conservation of energy:

$$
\frac{d}{d t} \int_{-\infty}^{\infty} \frac{1}{2} u(x, t)^{2} d x=0
$$

are satisfied for any differentiable solution to (1), but only one, namely mass (3) can be satisfied when the function $u(x, t)$ contains a shock. It is shown that requiring that mass $(3)$ be conserved defines the shock propagation. Splitting the real-line integration interval into $(-\infty,+\infty)=\lim _{s_{-}, s_{+} \rightarrow s}\left(-\infty, s_{-}\right) \cup\left(s_{+},+\infty\right)$, where $s_{-}(t)<s(t)<$ $s_{+}(t)$, the integral in (3) can be written in the limit for the case of a shock as:

$$
\frac{d}{d t}\left(\int_{-\infty}^{s_{-}(t)} u(x, t) d x+\int_{s_{+}(t)}^{\infty} u(x, t) d x\right)=\int_{-\infty}^{s_{-}(t)} u_{t}(x, t) d x+\int_{s_{+}(t)}^{\infty} u_{t}(x, t) d x-\left[s_{+}^{\prime} u\left(s_{+}, t\right)-s_{-}^{\prime} u\left(s_{-}, t\right)\right] .
$$

But from (1), we have $u_{t}=-\left(u^{2} / 2\right)_{x}$ in the integrals since $x \neq s$, so upon integration (5) becomes:

$$
-\left[\frac{1}{2} u^{2}\right]_{-\infty}^{\infty}+\left[\frac{1}{2} u^{2}\right]_{s_{-}}^{s_{+}}-s^{\prime}(t)[u]_{s_{-}}^{s_{+}} \equiv 0
$$

where the left hand side is set to zero because we require mass to be conserved. The first term is assumed to vanish, so solving for $s^{\prime}(t)$ yields the Rankine-Hugoniot condition for the speed of shock propagation:

$$
s^{\prime}(t)=\frac{1}{2}\left[u\left(s_{-}(t), t\right)+u\left(s_{+}(t), t\right)\right] \equiv \frac{1}{2}\left[u_{-}(t)+u_{+}(t)\right] .
$$

In this study, we are concerned with the energy integral in (4) and whether or not it is conserved during shock propagation. Because we required mass to be conserved for a shock resulting in (7), it turns out that the energy integral is not conserved. Following the same procedure outlined before yields the following expression for the rate of change of the energy integral $E(t) \equiv \int_{-\infty}^{\infty} \frac{1}{2} u(x, t)^{2} d x$ :

$$
E^{\prime}(t) \equiv \frac{d}{d t} \int_{-\infty}^{\infty} \frac{1}{2} u(x, t)^{2} d x=-s^{\prime}(t)\left[\frac{1}{2} u^{2}\right]_{s_{-}}^{s_{+}}+\left[\frac{1}{3} u^{3}\right]_{s_{-}}^{s_{+}}=\frac{1}{12}\left([u]_{s_{-}}^{s_{+}}\right)^{3}
$$

which is zero if and only if the function $u(x, t)$ is continuous. Hence, when wave breaking occurs and a shock starts to develop, the energy integral will start to change from its previously constant value. Equation (8) was analyzed in detail by Tran and Dritschel [13] for a representative initial profile $f(x)=-\sin x$.

In subsection 2.2, we analyze the asymptotic structure of $[u]_{s_{-}}^{s_{+}}$in detail to explicitly determine the energy change given in (8) shortly after the breaking time. Using this asymptotic structure, we define an alternative breaking criterion that can be used to obtain the breaking time given the energy curve $E(t)$.

\subsection{Shock Width}

The shock structure of the discontinuous solution to the Hopf equation is examined using asymptotic analysis for small times after the breaking time. In subsection 2.3, this result is used to determine the energy rate of change $E^{\prime}(t)$ for small times after the wave breaks.

It is more convenient to work with a moving coordinate system centered at the breaking point: $\zeta=\xi-\xi_{B}$, $y=x-u_{B} t-\xi_{B}$, and $w(y, t)=u(x, t)-u_{B}$, such that $y_{B}=\zeta_{B}=w_{B}=0$. In this moving frame, the solution to Hopf equation (2) becomes:

$$
\begin{array}{r}
w(y, t)=g(\zeta), \\
y=\zeta+w t,
\end{array}
$$


where $g(\zeta) \equiv f\left(\xi_{B}+\zeta\right)-u_{B}$.

We consider a small characteristic neighborhood of the breaking characteristic $\xi_{B}$ such that $|\zeta| \ll 1$. We have $g(0)=g^{\prime \prime}(0)=0$ (by definition and since breaking first occurs at an inflection point), so

$$
g(\zeta) \approx \zeta g^{\prime}(0)+\frac{1}{6} \zeta^{3} g^{(3)}(0)+\mathcal{O}\left(\zeta^{4}\right) \equiv a \zeta+b \zeta^{3}+\mathcal{O}\left(\zeta^{4}\right)
$$

For a positive breaking time $t_{B}=-1 / a$, we assume 0 to be a local minimum of $g^{\prime}$ so that $a<0$ and $b>0$ [4]. Calculated at the shock characteristics, we have $\zeta \rightarrow 0$ as $t \rightarrow t_{B}$ (since the shock width is zero upon the wave first breaking), so this approximation (10) is valid for $t-t_{B} \ll 1$, in a sense made more precise later, and ultimately allows one to obtain the local energy dissipation rate shortly after the breaking time.

Following Whitham [14], the shock structure in the moving frame $y=x-u_{B} t-\xi_{B}$ is determined by the solution of the following system:

$$
\begin{aligned}
\sigma(t)=\zeta_{L}+g\left(\zeta_{L}\right) t & =\zeta_{R}+g\left(\zeta_{R}\right) t, \\
\frac{1}{2}\left(g\left(\zeta_{L}\right)+g\left(\zeta_{R}\right)\right)\left(\zeta_{R}-\zeta_{L}\right) & =\int_{\zeta_{L}}^{\zeta_{R}} g(\zeta) \mathrm{d} \zeta
\end{aligned}
$$

where $\zeta_{L}<\zeta_{R}$ gives the two characteristics of $w(\zeta)$ connected by the shock located at $y=\sigma(t)$ in the moving frame. The first set of equations (11) comes from specifying (9) at the shock position $y=\sigma(t)$ and characteristics $\zeta=\zeta_{L}, \zeta_{R}$; the second comes from the Rankine-Hugoniot condition (7) of weak solutions to (1) or the so-called "equal-area rule" (see Whitham [14] for details). Solution of this system gives the shock trajectory $y=\sigma(t)$ and the shock characteristics $\zeta_{L}, \zeta_{R}$, which, using (11) allows us to compute the shock width $[u]_{s_{-}}^{s_{+}}=[w]_{\sigma_{-}}^{\sigma_{+}}$and finally the energy change rate in (8).

We solve the system (11)-(12) using approximation (10). Evaluating the right side of (12) gives $\int_{\zeta_{L}}^{\zeta_{R}} g(\zeta) \mathrm{d} \zeta \approx$ $a\left(\zeta_{R}^{2}-\zeta_{L}^{2}\right) / 2+b\left(\zeta_{R}^{4}-\zeta_{L}^{4}\right) / 4+\mathcal{O}\left(\zeta_{L}^{5}, \zeta_{R}^{5}\right)$. The left side gives $\frac{1}{2}\left[g\left(\zeta_{L}\right)+g\left(\zeta_{R}\right)\right]\left(\zeta_{R}-\zeta_{L}\right) \approx a\left(\zeta_{R}^{2}-\zeta_{L}^{2}\right) / 2+b\left(\zeta_{R}-\right.$ $\left.\zeta_{L}\right)\left(\zeta_{R}^{3}+\zeta_{L}^{3}\right)+\mathcal{O}\left(\zeta_{L}^{5}, \zeta_{R}^{5}\right)$, so equation (12) can be rewritten in this limit as:

$$
\frac{1}{4} b\left(\zeta_{R}^{4}-2 \zeta_{R}^{3} \zeta_{L}+2 \zeta_{R} \zeta_{L}^{3}-\zeta_{L}^{4}\right)=\frac{1}{4} b\left(\zeta_{L}+\zeta_{R}\right)\left(\zeta_{R}-\zeta_{L}\right)^{3} \approx \mathcal{O}\left(\zeta_{L}^{5}, \zeta_{R}^{5}\right)
$$

Since $\zeta_{L}<\zeta_{R}$, we find that:

$$
\zeta_{L} \approx-\zeta_{R}+\mathcal{O}\left(\zeta_{R}^{2}\right)
$$

Now, using (10), the second equation in (11), namely $\zeta_{R}-\zeta_{L}+t\left[g\left(\zeta_{R}\right)-g\left(\zeta_{L}\right)\right]=0$, can be rewritten as:

$$
\zeta_{R}-\zeta_{L}+t\left[a\left(\zeta_{R}-\zeta_{L}\right)+b\left(\zeta_{R}^{3}-\zeta_{L}^{3}\right)\right]=\left(\zeta_{R}-\zeta_{L}\right)\left[1+a t+b t\left(\zeta_{R}^{2}+\zeta_{R} \zeta_{L}+\zeta_{L}^{2}\right)\right] \approx \mathcal{O}\left(\zeta_{L}^{4}, \zeta_{R}^{4}\right) .
$$

Using (13), we have $\zeta_{R}-\zeta_{L} \approx 2 \zeta_{R}+\mathcal{O}\left(\zeta_{R}^{2}\right)$ and $\zeta_{R}^{2}+\zeta_{R} \zeta_{L}+\zeta_{L}^{2} \approx \zeta_{R}^{2}+\mathcal{O}\left(\zeta_{R}^{3}\right)$, so (11) reduces to:

$$
\zeta_{R}^{2} \approx-(1+a t) / b t+\mathcal{O}\left(\zeta_{R}^{3}\right)
$$

Hence, we obtain:

$$
\zeta_{R} \approx \sqrt{-\frac{1+a t}{b t}}+\mathcal{O}\left(\zeta_{R}^{2}\right) \approx \sqrt{\frac{t-t_{B}}{b t_{B}^{2}}}+\mathcal{O}\left(t-t_{B}\right), \quad t \geq t_{B} .
$$

The positive root was chosen so that $\zeta_{L}<\zeta_{R}$ from (13). From (14), the approximation (10) being valid for $\zeta_{R} \ll 1$ translates to being valid for small times $t-t_{B}>0$ after the breaking time $t_{B}=-1 / f^{\prime}\left(\xi_{B}\right)$ such that $t-t_{B} \ll f^{(3)}\left(\xi_{B}\right) /\left[6 f^{\prime}\left(\xi_{B}\right)^{2}\right]$, since $b \equiv f^{(3)}\left(\xi_{B}\right) / 6$.

Using the result (14), we can now calculate the shock width $[u(x, t)]_{x=s_{-}}^{x=s_{+}}=\left[u_{B}+w(y, t)\right]_{y=\sigma_{-}}^{y=\sigma_{+}}=[g(\zeta)]_{\zeta=\zeta_{L}}^{\zeta=\zeta_{R}}$ that develops during a short time after wave breaking. First, in (11), we note that $\sigma(t)=\mathcal{O}\left(\zeta_{R}^{2}\right)$, since $\sigma(t)=$ $\zeta_{R}+t g\left(\zeta_{R}\right)=\zeta_{L}+t g\left(\zeta_{L}\right) \approx-\left[\zeta_{R}+t g\left(\zeta_{R}\right)\right]+\mathcal{O}\left(\zeta_{R}^{2}\right)=\mathcal{O}\left(\zeta_{R}^{2}\right)$ by antisymmetry. Thus, solving (11) gives $g\left(\zeta_{L}\right) \approx$ $-\zeta_{L} / t+\mathcal{O}\left(\zeta_{R}^{2}\right) \approx+\zeta_{R} / t+\mathcal{O}\left(\zeta_{R}^{2}\right)$ and $g\left(\zeta_{R}\right) \approx-\zeta_{R} / t+\mathcal{O}\left(\zeta_{R}^{2}\right)$. Thus, we obtain the asymptotic shock width shortly after the breaking time $t_{B}=-1 / f^{\prime}\left(\xi_{B}\right)$ :

$$
[u]_{s_{-}}^{s_{+}} \approx-2 \zeta_{R} / t+\mathcal{O}\left(\zeta_{R}^{2}\right) \approx-2 \sqrt{\frac{t-t_{B}}{b t_{B}^{4}}}+\mathcal{O}\left(t-t_{B}\right), \quad t \geq t_{B} .
$$

This quantity, of course, is zero for $0 \leq t \leq t_{B}$. It is important to note that this function is non-analytic at $t=t_{B}$. That this is so forms a basis for a new breaking criterion. 


\subsection{Energy Dissipation}

Using the asymptotic results obtained in subsection 2.2 , it is shown that the energy $E(t)$ decreases, where $E^{\prime}(t)$ in (8) describes energy dissipation. Asymptotic results for the shock width $[u]_{s_{-}}^{s_{+}}$in (15) show that the energy dissipation rate $E^{\prime}(t)$ has a singular second derivative. It is this non-analytic structure of the energy dissipation rate at the breaking point that will suggest a new method for numerical computation of the breaking time as the location of this singularity.

Combining (8) and (15) yields the following asymptotic result for the energy derivative:

$$
E^{\prime}(t) \approx-\frac{2}{3 b^{3 / 2} t_{B}^{6}}\left(t-t_{B}\right)^{3 / 2}+\mathcal{O}\left(\left(t-t_{B}\right)^{2}\right), \quad t \geq t_{B} .
$$

For $0 \leq t \leq t_{B}$, the energy dissipation rate is zero.

This result shows that the energy rate of change is negative, so upon wave breaking, the total energy (4) starts to decrease. Stoker [10] attributes this effect in the nonlinear shallow-water equations as analogous to the onset of turbulent dissipation that occurs in bore propagation in water waves. In addition, we observe that this function is non-analytic, which is not obvious if one only considers (8) without studying in more detail the shock width (15). To see the consequences of this singular nature of the energy function near the breaking time, we compute the third derivative $E^{(3)}(t)$ :

$$
E^{(3)}(t)=\left\{\begin{array}{lr}
0 & 0<t<t_{B} \\
\frac{-1}{2 b^{3 / 2} t_{B}^{6}}\left(t-t_{B}\right)^{-1 / 2} & 0<t-t_{B} \rightarrow 0 .
\end{array}\right.
$$

This function is shown in Figure 1.

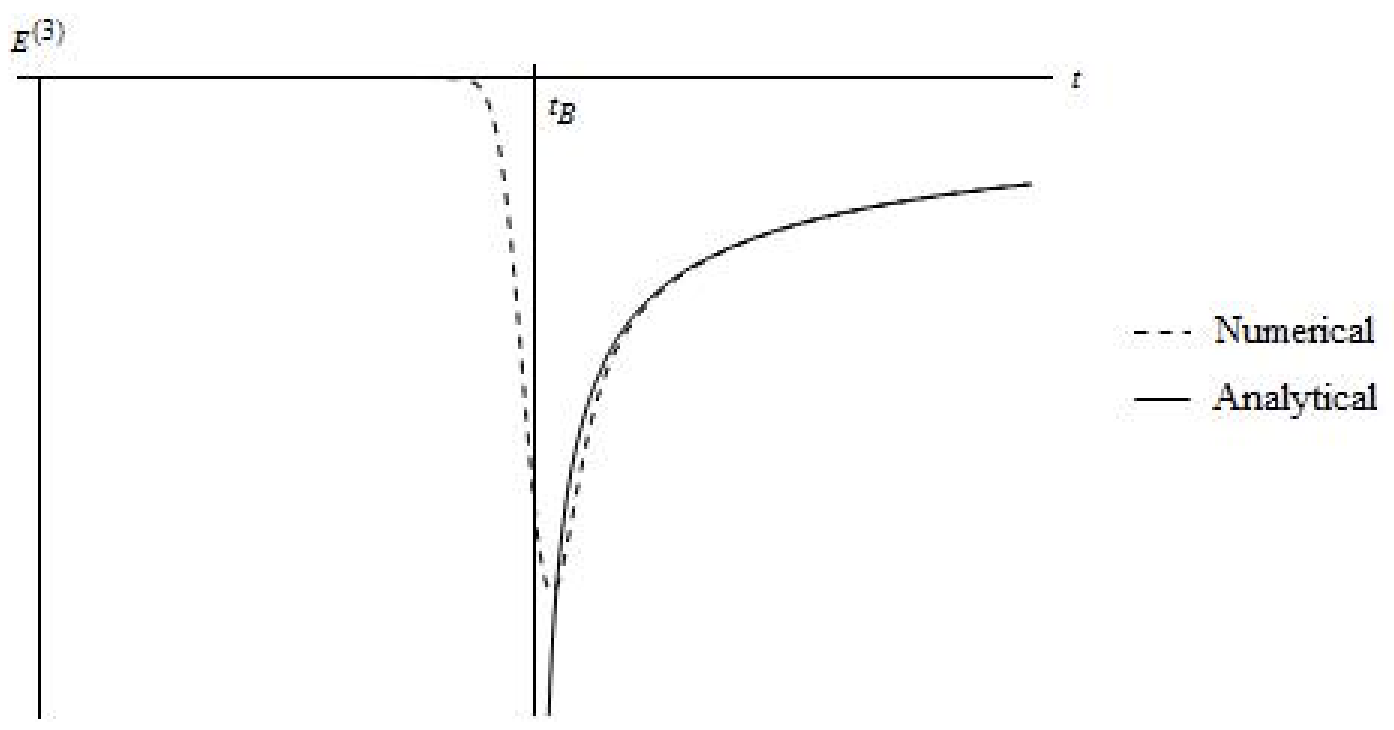

Figure 1: Third derivative of the energy and sketch of smoothed numerical analogue.

Clearly, the energy derivative $E^{(3)}(t)$ jumps from zero to a singularity at $t=t_{B}$. Given that this function is continuous (namely, zero) for $0<t<t_{B}$, this drastic change in behavior motivates a redefinition of the breaking time that can be readily applied given the analytical form of the energy curve:

The breaking time $t_{B}$ is the smallest time $t_{B}>0$ at which $E^{(3)}(t)$ displays a singularity.

This is an analytical breaking time criterion that requires an exact form of the curve $E^{(3)}(t)$ to work. In practice, obtaining $E(t)$ numerically introduces artificial smoothing into the non-analytic energy distribution, particularly if a finite-difference method is used to solve the Hopf equation (1). A sketch of a smoothed curve representative of such a numerically obtained distribution is juxtaposed along with the analytical curve (17) in Figure 1. In such a case, the singularity will be tempered and instead display itself as a prominent local minimum. Because mesh refinement reduces the amount of artificial diffusion introduced by a finite difference method [7], this prominent 
minimum would be expected to converge to the singularity upon refinement. Thus, we define a numerical breaking time criterion to use when the energy curve $E(t)$ is obtained numerically:

The numerical breaking time $t_{B}$ is the smallest time $t_{B}>0$ at which $E^{(3)}(t)$ displays a local minimum.

This is the main theoretical result of this paper. Its applicability is verified in section 3 for a numerical case study.

\subsection{Moving Averages}

The numerical integration of the steep wavefront employed in the following section's numerical procedure introduces significant error, so numerically differentiating this distribution three times as required by the breaking criterion generates large fluctuations in the derivative distribution. A smoothing method is developed to compensate for this problem. The following two observations are made concerning the form of this error: (i) the error is more or less distributed randomly above and below the unperturbed optimal energy distribution, and (ii) the error distribution varies significantly between each grid point on the time axis. Using observations (i) and (ii), we can (i) model the error distribution analytically using a sinusoid proxy curve and (ii) use as the sinusoidal wavelength the time discretization scale $\Delta t$ (a more careful and less convenient argument might use a statistical formulation rather than sinusoids, but we suspect that the results would be equivalent). It is shown that this error distribution can be made negligible upon mesh refinement by replacing the perturbed energy distribution with its "locally averaged" counterpart.

A unique feature of this problem is that the perturbations vary on the scale of the time discretization. The advantage of utilizing a custom smoothing method is that, as the time discretization is refined, the smoothed function converges to the desired unperturbed curve. This convergence occurs as a result of formulating the averaging window to vanish with the numerical scheme's time discretization. As the discretization is refined, any negative effects caused by the smoothing vanish along with the undesired perturbations. This being said, the method here is certainly open to further theoretical and computational refinement using the existing formalism developed in e.g. [8].

This situation is formulated in terms of smooth functions. Let the energy distribution obtained from the following section's integration procedure be:

$$
E(t)=E_{0}(t)+a_{0} \sin (k t+\phi),
$$

where $E_{0}(t)$ is the unperturbed (i.e. exact) smooth energy curve, $a_{0}$ is the characteristic amplitude of the disturbance, $k=k_{0} / \Delta t$ is the characteristic wavenumber defined on the scale of the time domain discretization, and $\phi$ is a phase factor. Differentiating this curve three times as required for determining the breaking criterion gives:

$$
E^{(3)}(t)=E_{0}^{(3)}(t)-a_{0} k^{3} \cos (k t+\phi) .
$$

Evidently, as the mesh is refined (or $\Delta t \rightarrow 0$ ), the error term dominates the third derivative of the energy function (19) as $k \rightarrow \infty$. This necessitates the development of a smoothing method.

The goal is to make the oscillatory perturbation term disappear by applying to $E^{(3)}(t)$ some "averaging opera-

tion" so that $E_{0}^{(3)}(t)$ is recovered from $E^{(3)}(t)$. We define an averaging operator $A[].(t)$ that integrates the argument function over a small interval $2 \Delta$ centered at time $t$. The interval $2 \Delta$ should be sufficiently large that rapidly oscillatory integrands will cancel but small enough to preserve the form of a slowly varying argument function; it should also depend on the oscillation wavenumber $k=k_{0} / \Delta t$ as $k \rightarrow \infty$ to maintain this effect. Using this sinusoidal model for the error distribution, one can quantify how small or large to make this integration interval. Let the action of the averaging operator $A$ on a function $f(t)$ be:

$$
A[f](t)=\frac{1}{2 \Delta} \int_{-\Delta}^{\Delta} f(t+y) d y
$$

This linear operator can be applied iteratively using a recursive definition: $A^{1}[f](t)=A[f](t)$, and $A^{n}[f](t)=$ $A\left[A^{n-1}[f]\right](t)$ for $n>1$. It should be noted that if $f(t)$ is defined on the time interval $I_{0}=[0, T]$, then the averages $A^{n}[f](t)$ are defined on the smaller time intervals $I_{n}=[n \Delta, T-n \Delta]$ due to the nonlocal nature of the operator $A$; thus, the $n$-iterated average is well defined only for $n<T / 2 \Delta$; this is relevant in the numerical implementation of the averaging procedure. Finally, although using more averaging applications smooths the argument function 
out more, computational cost and "over-smoothing" motivate us to seek a minimal number of applications $n$ of the averaging operator that will diminish the error distribution in the limit $k \rightarrow \infty$.

We define conditions ultimately required on the integration half-interval $\Delta$ and the minimum number of averages $n$ so that the following convergence criterion is satisfied for the averaged energy third derivative as the perturbation wavelength vanishes:

$$
\lim _{k \rightarrow \infty} \sup _{t \in I_{n}}\left|A^{n}\left[E^{(3)}\right](t)-E_{0}^{(3)}(t)\right|=0 .
$$

This condition requires that we recover our desired energy distribution $E_{0}(t)$ from the averaged distribution $A^{n}[E](t)$ as the wavelength $\Delta t$ for the numerical error perturbations vanishes. Using the form of $E^{(3)}$ given in (19), the linearity of the averaging operator and applying the triangle inequality to the absolute value gives the following two sufficient conditions that fulfill (21):

$$
\begin{aligned}
& \lim _{k \rightarrow \infty} \sup _{t \in I_{n}}\left|A^{n}\left[E_{0}^{(3)}\right](t)-E_{0}^{(3)}(t)\right|=0, \\
& \lim _{k \rightarrow \infty} k^{3} \sup _{t \in I_{n}}\left|A^{n}[\cos (k .+\phi)](t)\right|=0 .
\end{aligned}
$$

In this limiting regime, the first condition (22) requires that applying the averaging procedure to the smooth and slowly varying function $E_{0}^{(3)}(t)$ gives that of the identity, while the second condition (23) requires that the rapidly oscillating sinusoid of large amplitude $k^{3}$ vanish when the averaging is applied. In practice, the perturbation wavelength $\Delta t$ is nonzero but small, so satisfaction of these two conditions recovers $E_{0}^{(3)}$ from $A^{n}\left[E^{(3)}\right]$ to a good approximation.

Conditions (22) and (23) are evaluated to determine requirements on $\Delta$ and $n$. It can be shown by induction that the suprema in (22) and (23) satisfy the following inequalities:

$$
\begin{aligned}
& \sup _{t \in I_{n}}\left|A^{n}\left[E_{0}^{(3)}\right](t)-E_{0}^{(3)}(t)\right| \leq C_{n} \Delta^{2}, \quad C_{n}=\frac{n}{6} \sup _{t \in I_{0}}\left|E_{0}^{(5)}(t)\right|, \\
& k^{3} \sup _{t \in I_{n}}\left|A^{n}[\cos (k .+\phi)](t)\right| \leq k^{3} a_{n}, \quad a_{n}=a_{0}(\sin (k \Delta) / k \Delta)^{n} .
\end{aligned}
$$

Thus, the first condition (22) requires that $\Delta \rightarrow 0$ as $k \rightarrow \infty$, and the second condition (23) requires that (a) $k \Delta \rightarrow \infty$ and (b) $k^{3-n} / \Delta^{n} \rightarrow 0$ as $k \rightarrow \infty$. If we set $\Delta \equiv k^{-m}$, then these conditions require that $0<m<1$ and $3-n+n m<0$.

To summarize, in terms of the perturbation wavelength $\Delta t$, the averaging procedure applied to the third energy derivative is characterized by the following integration half-interval $\Delta$, minimum number of averages $n$, and averaging error:

$$
\begin{aligned}
& \Delta=(\Delta t)^{m}, \quad 0<m<1 \\
& n>\frac{3}{1-m}, \\
& \sup _{t \in I_{n}}\left|A^{n}\left[E_{0}^{(3)}\right](t)-E_{0}^{(3)}(t)\right|=\mathcal{O}\left(n \Delta^{2}\right), \quad I_{n}=[n \Delta, T-n \Delta] .
\end{aligned}
$$

The perturbation amplitude in $E^{(3)}$ after averaging decreases from $\mathcal{O}\left((\Delta t)^{-3}\right)$ to $\mathcal{O}\left((\Delta t)^{n-n m-3}\right)$ as $\Delta t \rightarrow 0$. Increasing the parameter $m$ requires more averages but reduces the overall averaging error as the time discretization is refined; if $m=1 / 2$, for example, then the method requires $n=7$ averages and yields $\mathcal{O}(\Delta t)$ convergence of the averaging error. The perturbation amplitude in this case is $\mathcal{O}\left((\Delta t)^{1 / 2}\right)$ as $\Delta t \rightarrow 0$.

\section{Application to Gaussian Wave}

In this section, a particular initial wave is studied using the methodology outlined in section 2 to serve as illustration of the approach and as verification that the analytical theory is consistent. 


\subsection{Numerical Details}

The following initial wave profile $u(x, 0)=f(x)$ is used for the numerical solution of Hopf equation (1):

$$
f(x)=\exp \left(-18(x-1)^{2}\right) .
$$

This Gaussian of wavelength 1 has amplitude 1 and is centered at $x=1$. Such a profile is representative of an isolated tsunami water wave, for example. The exact breaking time for this profile is $t_{B}=e^{1 / 2} / 6 \approx 0.274787$.

The numerical details used in computations are briefly given. The computational spatial interval $x:[0,2+T]$ and time interval $t:[0, T]$ are discretized into $J$ and $K$ increments respectively: $x_{j}=j \Delta x, j \in\{0,1, \ldots J\}$ and $t_{k}=k \Delta t, k \in\{0,1, \ldots K\}$, where $\Delta x=(2+T) / J$ and $\Delta t=T / K$. Function $u(x, t)$ is evaluated at discrete points on the space-time grid $\left(x_{j}, t_{k}\right)$. Computations were run for total time $T=2 t_{B}$.

The Hopf equation (1) upon discretization was solved using the Centered Flux-Limiter method developed by Toro [12] that hybridizes the FORCE and Richtmyer numerical fluxes yielding a second-order accurate TVD numerical method; the Superbee flux limiter was used (see [12] for details). Transmissive boundary conditions were used at endpoints of the computational spatial domain.

The energy function $E(t)$ in (4) was obtained by numerically integrating the function data $u\left(x_{j}, t_{k}\right)$ using Simpson's rule quadrature. Next, the third derivative $E^{(3)}(t)$ was obtained by numerically differentiating the energy data $E\left(t_{k}\right)$. Then, the averaging method in section 2.4 was applied to $E^{(3)}(t)$ for $\Delta=(\Delta t)^{3 / 4}$ and $n=13$. Finally, application of the numerical criterion for breaking introduced in section 2.3 required finding the smallest time $t_{m}$ at which the function data set achieved a local minimum.

Computations were performed on a laptop with 4 GB RAM and a $2.13 \mathrm{GHz}$ processor. The longest computation (solving the differential equation) took 23 minutes for $J=2500$.

\subsection{Results}

The numerical solution of Hopf equation (1) and initial condition (25) is compared with the exact analytical solution (2) given in implicit form in Figure 2. The two functions give good agreement until the occurrence of the breaking time, at which the numerical solution presents a shock profile while the analytical solution becomes multivalued.

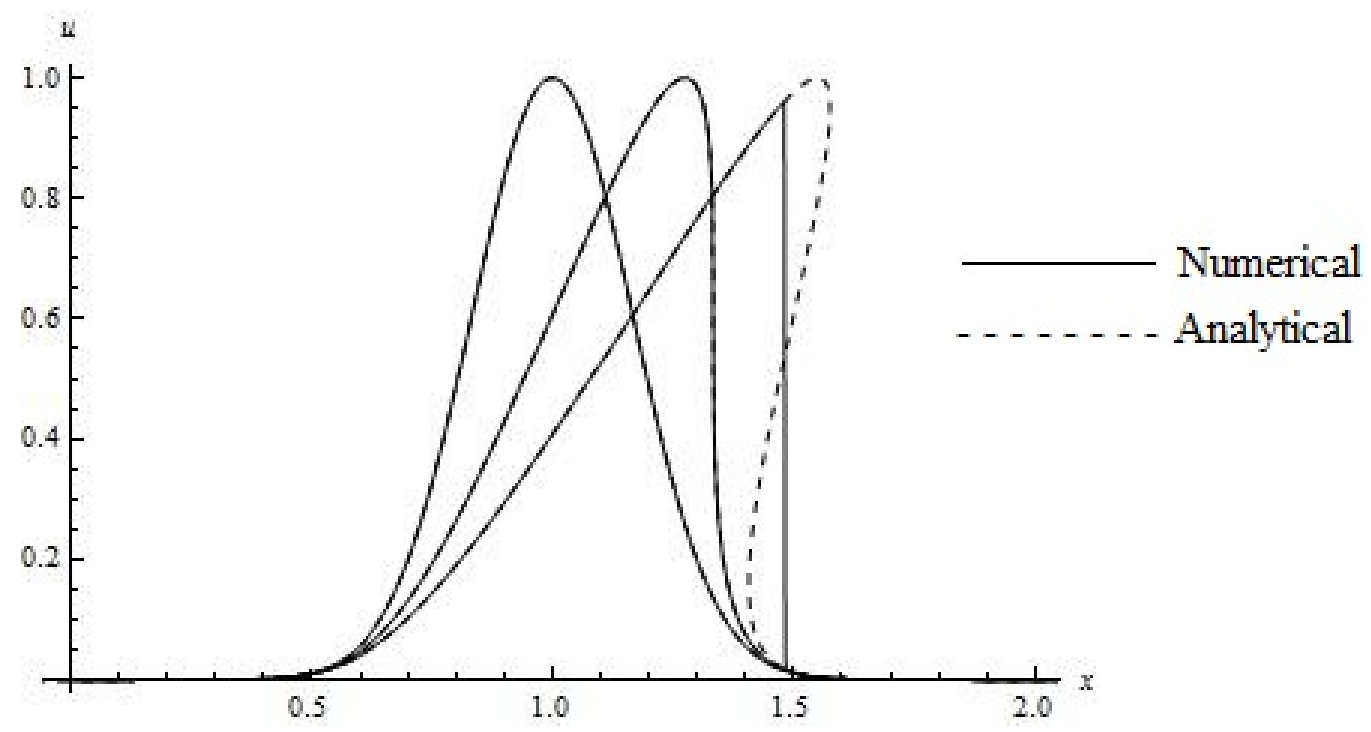

Figure 2: Comparison of analytical solution to numerical solution $(J=2500)$. Plotted at times $t=0, t_{B}, 2 t_{B}$.

The energy function (4) is shown in Figure 3. Evidently, it maintains a constant value until a time near the breaking time, at which point it starts to decrease, in agreement with the analytical result (16). 


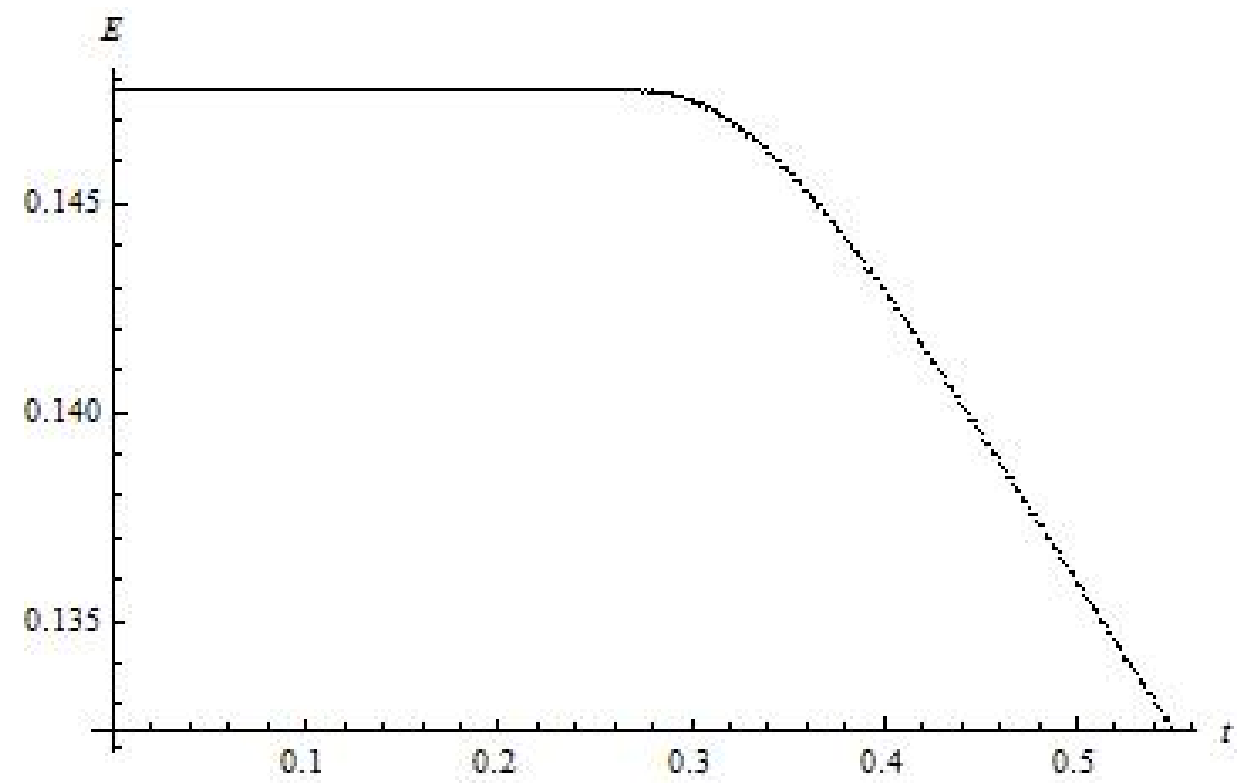

Figure 3: Plot of energy function $(J=2500)$.

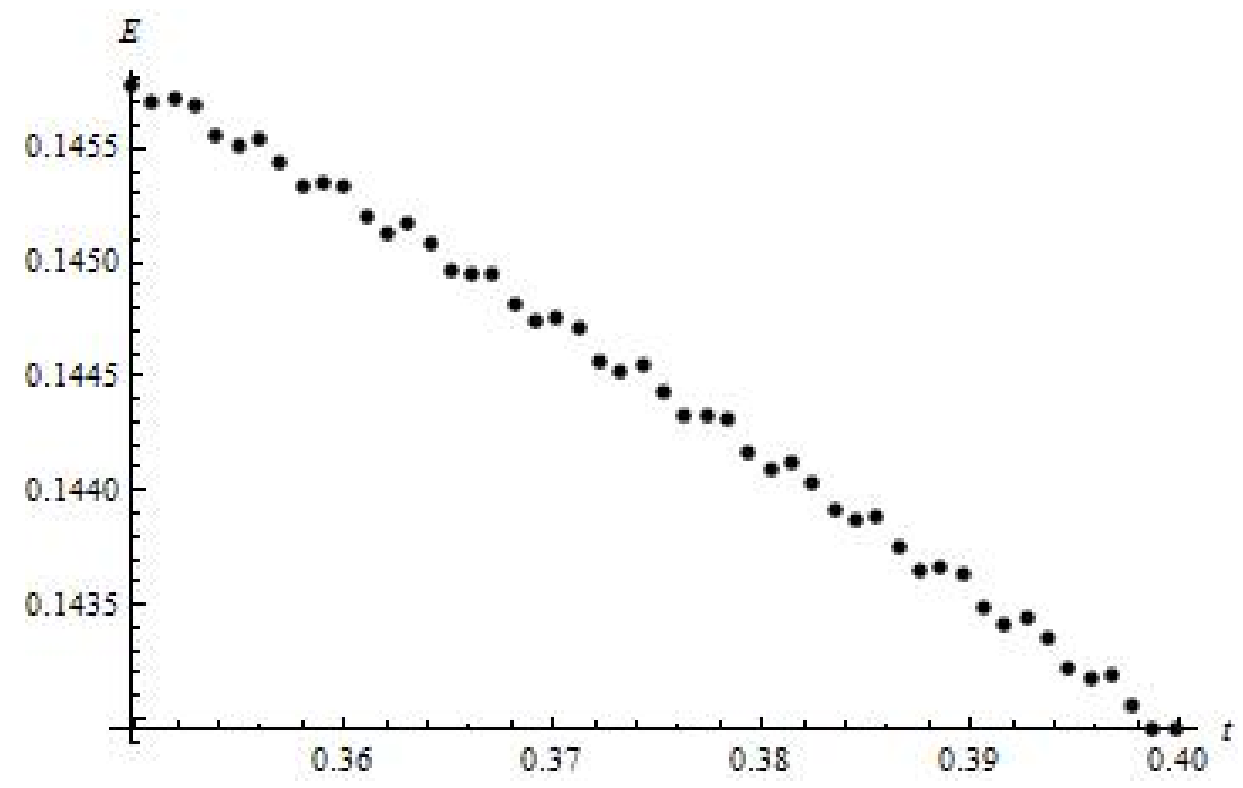

Figure 4: Close-up of energy curve in Figure 3.

To see why the smoothing method employed in section 2.4 is necessary, a close-up of the energy curve is shown in Figure 4. Evidently, small perturbations from the monotonically decreasing energy profile exist on the scale of each grid point. 


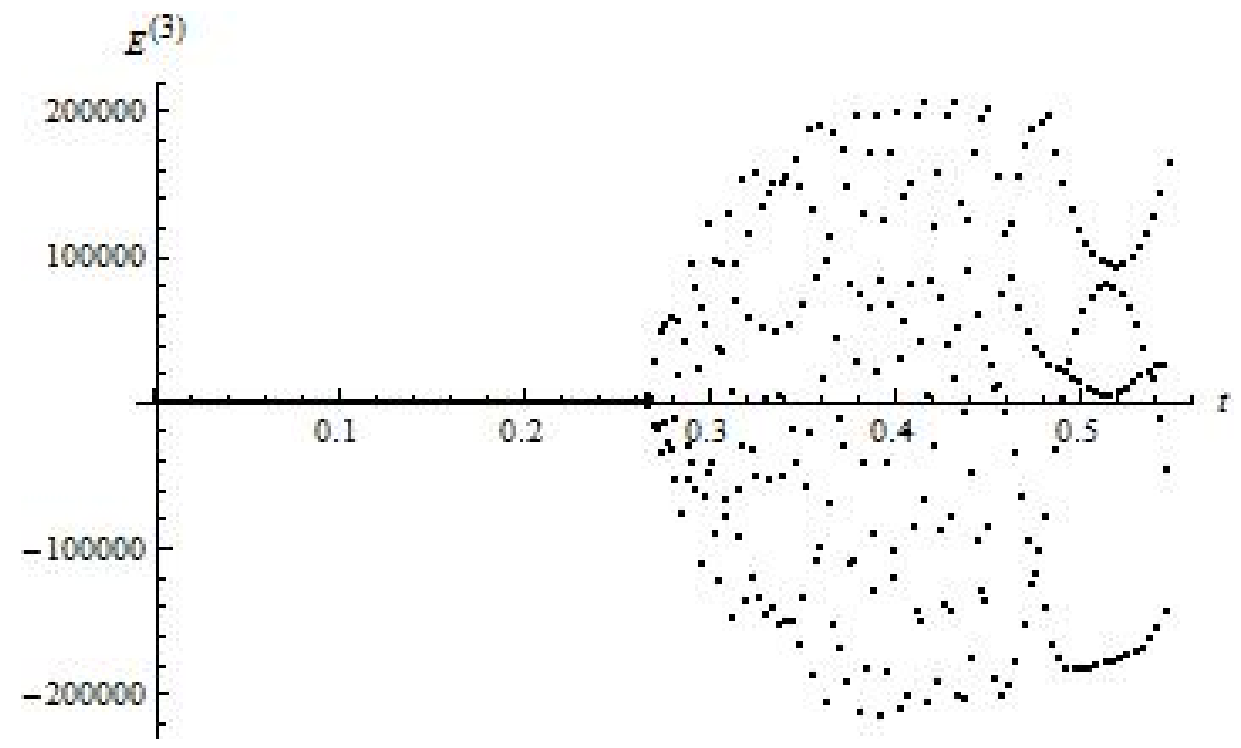

Figure 5: Plot of energy third derivative $(J=2500)$.

The effect these small perturbations have on the third derivative, shown in Figure 5, is much larger. The fluctuations make analysis of this curve impossible. It is presumed that this error is due to numerical integration of the wave after it has broken and become very steep, as in Figure 2.

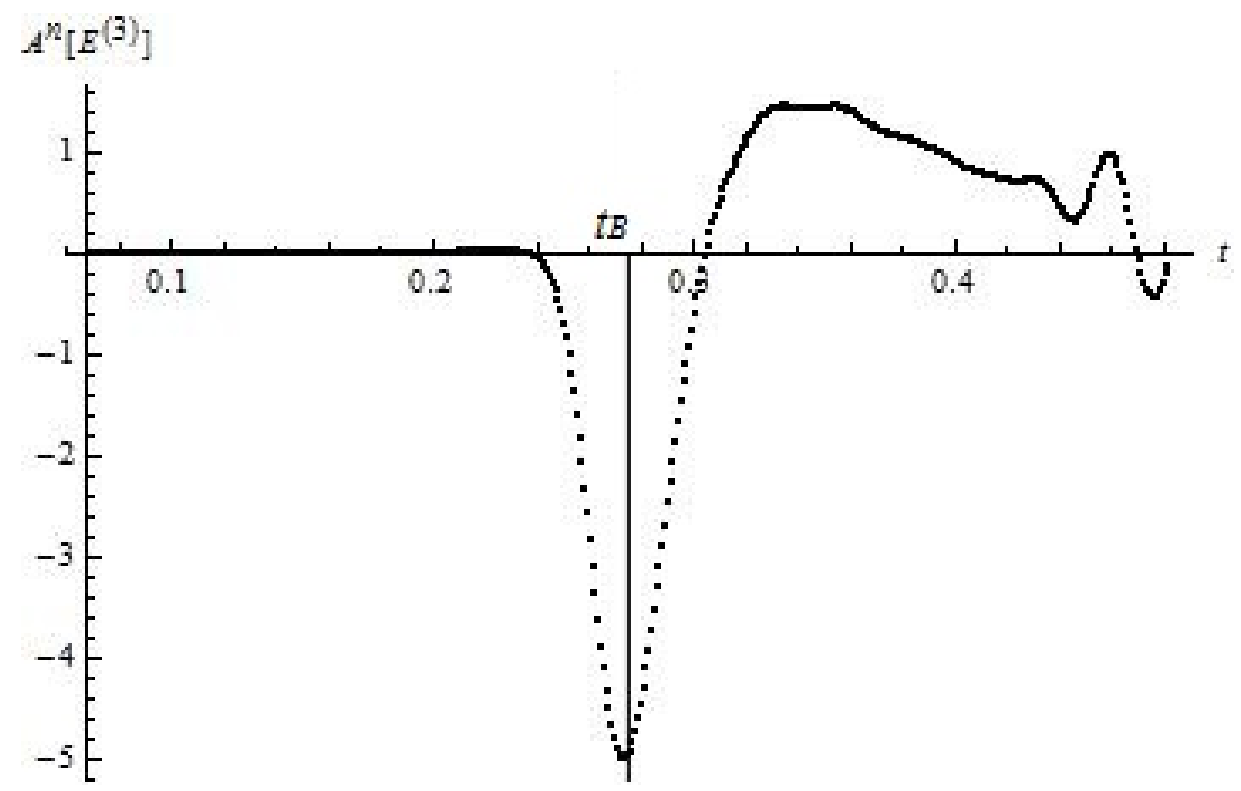

Figure 6: Averaged third derivative $(J=2500, m=3 / 4, n=13)$.

The averaged version of this curve is shown in Figure 6. Clearly, the oscillations have been greatly tempered, and the true function's profile is much more visible. In particular, a prominent local minimum is evident near the breaking time. In terms of the analytical result (17), this minimum corresponds to a tempered and smooth version of the singularity in the energy function's third derivative. 


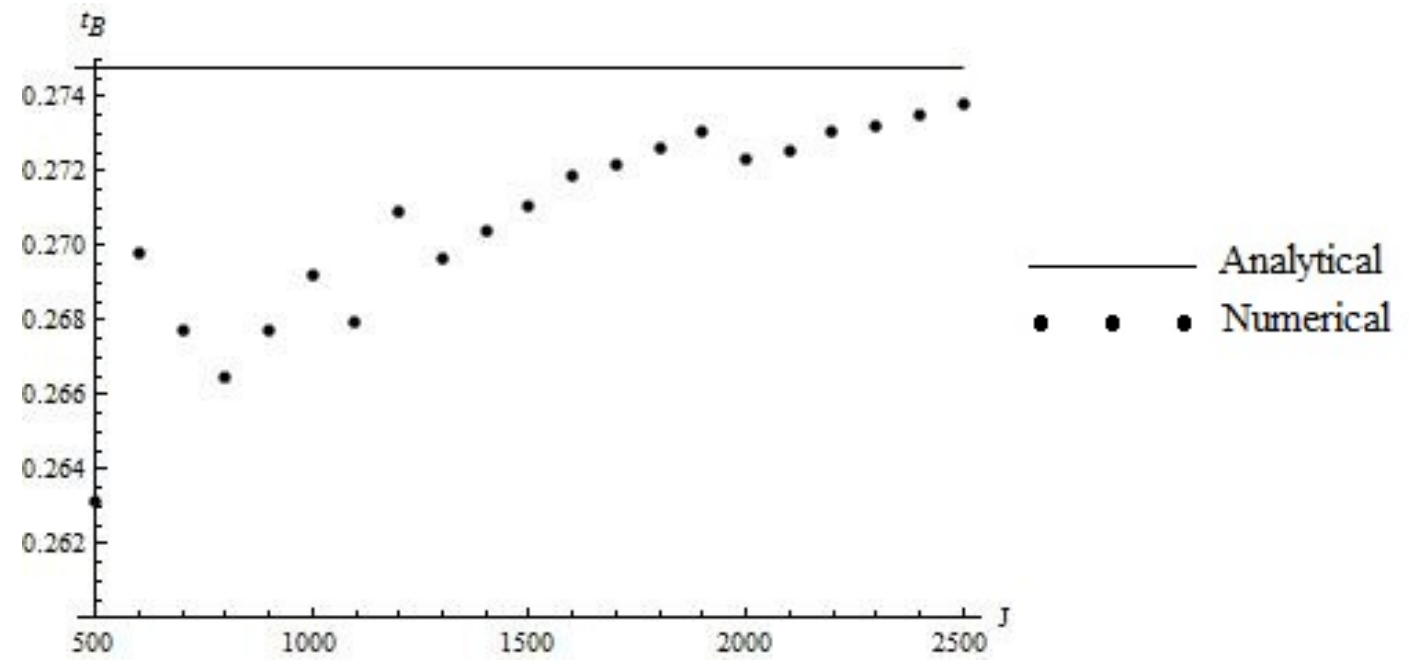

Figure 7: Numerical breaking times. Horizontal line indicates analytical breaking time.

The location of this minimum on the time axis has been computed for various spatial step numbers $J$ from $J=500$ to $J=2500$ in steps of 100 . The results are juxtaposed with the exact value of the breaking time in Figure 7. Evidently, as the mesh is refined, the numerical results give better agreement overall. Although convergence is not completely monotonic, the accuracy visibly increases with mesh refinement, such that for $J=2500$, the computed time is within $0.5 \%$. However, the convergence is rather slow and could undoubtedly benefit from sequence acceleration (see e.g. [1]).

\section{Summary of Results}

In this study, a new approach was described for obtaining the time of nonlinear wave breaking in solutions to the Hopf equation (1). The motivation for the method comes from an asymptotic result derived in section 2.3 that shows that the energy function undergoes a singular transition at the breaking time that is clearly visible in the function's third derivative. A breaking criterion was formulated and tested numerically in section 3 for a Gaussian wave profile. To account for numerical error fluctuating on the scale of the discretization that would otherwise obscure the energy function's third derivative, an averaging method was developed in section 2.4 that successfully lessened the magnitude of the error perturbations and recovered a truer approximation to the ideal curve. The numerical predictions for the breaking time were shown to improve as the numerical discretization was refined, verifying that the analytical ideas are consistent.

There are several possible applications of these results. First, this approach might be applied to other interesting one-dimensional systems that also exhibit nonlinear wave breaking and shock formation, such as the nonlinear shallow-water equations or the equations of gas dynamics. For these systems, the asymptotic results may parallel those in section 2.2 given that similar formalisms for the Rankine-Hugoniot conditions exist [7]; moreover, finitedifference methods for these equations' numerical solution are already well-developed [12]. In addition, the possibility exists for an application to two- and three-dimensional analogues of the Hopf equation such as the Euler equations of inviscid fluid dynamics. For these more realistic equations, the time of nonlinear wave-breaking is instead associated with the onset of turbulence. If this energy-based criterion for nonlinear blowup also applies to this three-dimensional situation, the approach given here could lead to alternative methods of isolating and studying the time of turbulence onset.

\section{Acknowledgments}

This research was supported by the California State University Council on Ocean Affairs, Science and Technology (COAST). It was also co-funded by the Department of Defense and NSF (award DMS-1156612). The author is 
grateful for the contributions of Tucker Hartland and Mark Brandao to the project. The author appreciates the advising and support of Dr. Sergei Fomin.

\section{References}

[1] Bender, C. M., \& Orszag, S. A. Advanced Mathematical Methods for Scientists and Engineers. McGrewHill, New York, NY, 1978.

[2] Chugunov, V., Fomin, S., \& Shankar, R. (2014). Influence of underwater barriers on the distribution of tsunami waves. Journal of Geophysical Research: Oceans, 119(11), pp. 7568-7591.

[3] Constantin, P. (2007) On the Euler equations of incompressible fluids, Bull. Amer. Math. Soc. (N.S.) 44, pp. 603-621.

[4] Dubrovin, B., \& Elaeva, M. (2012) On the critical behavior in nonlinear evolutionary PDEs with small viscosity, Russ. J. Math. Phys., 19(4), pp. 449-460.

[5] Jeffrey, A., \& Russo, G. (1987) Comparison of theoretical and numerical criteria for water wave breaking, Wave motion, 9(3), pp. 261-268.

[6] Klein, C., \& Roidot, K. (2013) Numerical study of shock formation in the dispersionless Kadomtsev-Petviashvili equation and dispersive regularizations, Phys. D., 265, pp. 1-25.

[7] LeVeque, R. J., \& Le Veque, R. J. Numerical methods for conservation laws (Vol. 132), Birkhuser, Basel, 1992.

[8] Li, Q., \& Racine, J. S. Nonparametric econometrics: Theory and practice, Princeton University Press, 2007.

[9] Shankar, R., Sheng, Y., Golbek, M., Hartland, T., Gerrodette, P., Fomin, S., \& Chugunov, V. (2015). Linear long wave propagation over discontinuous submerged shallow water topography. Appl. Math. Comput. 252, pp. 27-44.

[10] Stoker, J. J. (1957), Water Waves: The Mathematical Theory With Applications, Interscience Publishers Inc., N. Y.

[11] Titov, V. V., \& Synolakis, C. E. (1995) Modeling of breaking and nonbreaking long-wave evolution and runup using VTCS-2, Journal of Waterway, Port, Coastal, and Ocean Engineering, 121(6), pp. 308-316.

[12] Toro, E. F. Riemann solvers and numerical methods for fluid dynamics (Vol. 16), Springer, Berlin, 1999.

[13] Tran, C. V., \& Dritschel, D. G. (2010) Energy dissipation and resolution of steep gradients in one-dimensional Burgers flows. Physics of Fluids 22(3), pp. 37-102.

[14] Whitham, G. B. (1974), Linear and Nonlinear Waves, John Wiley, New York. 